\title{
Acesso, acolhimento e acompanhamento: três desafios para o cotidiano da clínica em saúde mental*
}

Moema Belloni Schmidt

Ana Cristina Figueiredo

O presente artigo toma a avaliação da qualidade de serviços de saúde mental como um instrumento permanente de reflexão sobre a clínica nesses serviços para além de um projeto de avaliação a priori feito de fora, para qualificar de dentro o trabalho da atenção psicossocial, a partir do que aparece durante ou a posteriori. Apontamos eixos do campo da avaliação que podem ser aplicados tanto na análise quanto na organização do cotidiano da clínica nos CAPS. Propomos os eixos acesso, acolhimento e acompanhamento como elementos fundamentais para a análise da assistência e para a qualificação permanente dos serviços. Apresentamos aqui parte de um estudo de caso de teor avaliativo realizado no CAPS Profeta Gentileza - SMS-Rio no ano de 2006, para tese de doutoramento de uma das autoras, que obteve material dos casos através de relato gravado em reunião de supervisão de equipe no serviço. A supervisão foi escolhida como lócus privilegiado da pesquisa por ser considerada um dispositivo fundamental para o funcionamento do serviço, a organização e a dinâmica do trabalho em equipe, e funcionar como um avaliador permanente do cotidiano do CAPS.

Palavras-chave: Atenção psicossocial, avaliação de serviços, saúde mental, supervisão

* Este texto foi elaborado a partir do trabalho original apresentado no Simpósio de Saúde Mental no III Congresso Internacional e XIX Congresso Brasileiro de Psicopatologia Fundamental, 4 a 7 de setembro de 2008, Niterói, RJ.

O texto baseia-se também em pesquisa de doutoramento realizada pela primeira autora que envolveu o CAPS Profeta Gentileza - SMS-Rio. Parte da pesquisa ocorreu nas reuniões de supervisão clínica sob a responsabilidade da segunda autora. 
O que apresentamos a seguir é parte de um estudo de caso de teor avaliativo realizado no CAPS Profeta Gentileza ${ }^{1}$ no ano de 2006, para tese de doutoramento de uma das autoras (cf. Schmidt, 2007), que obteve material dos casos através de relato gravado em reunião de supervisão de equipe no serviço. A supervisão ${ }^{2}$ foi escolhida como lócus privilegiado da pesquisa por ser considerada um dispositivo fundamental para o funcionamento do serviço, a organização e a dinâmica do trabalho em equipe, e funcionar como um avaliador permanente do cotidiano do CAPS. Este estudo aponta eixos do campo da avaliação que podem ser aplicados tanto na análise quanto na organização do cotidiano da clínica na atenção psicossocial. Propomos os três eixos - acesso, acolhimento e acompanhamento - como norteadores do trabalho ao mesmo tempo que são elementos fundamentais para a análise da assistência e para a qualificação permanente dos serviços. Esses eixos estão presentes nos níveis macro, médio e micro da avaliação da qualidade em saúde. Situamos brevemente cada um:

- Acesso: Donabedian (1973) optou pelo uso do termo acessibilidade, definindo-o como um dos aspectos da oferta do serviço em relação à capacidade de responder às demandas. Sendo assim, é mais abrangente do que a simples oferta de serviços de saúde, mas refere-se às características do serviço e dos recursos de saúde que facilitam ou limitam seu uso por potenciais usuários. Destacamos que em seu aspecto organizacional, que remete ao desenho do serviço, o acesso está diretamente atrelado ao acolhimento. Neste caso, ambos são inseparáveis.

- Acolhimento: é o primeiro contato com quem nos procura, a partir do qual será definida toda a sequência de atendimentos, é "a abertura do jogo de xadrez" como nos ensina Freud. É o momento em que todos os recursos do serviço são postos à prova: quem atende; o que pode oferecer; como pode acolher, avaliar e discriminar a demanda. Silva Júnior \& Mascarenhas (2004) destacam três dimensões do acolhimento: postura, técnica e

1. O CAPS Profeta Gentileza fica situado no bairro de Inhoaíba, entre os bairros de Campo Grande e Santa Cruz, na zona oeste do município do Rio de Janeiro.

2. A supervisão é um dispositivo de suporte clínico-institucional do CAPS e acontece com regularidade semanal nas reuniões de equipe. A supervisora do CAPS Profeta Gentileza é a professora e psicanalista Ana Cristina Figueiredo desde março de 2004. 
princípio de reorientação do serviço. A primeira dimensão (postura) implica a atitude humanizada e receptiva dos profissionais ao receber, escutar e tratar as demandas dos usuários; implicando relação de interesse mútuo, confiança e apoio. Esta dimensão abrange tanto as relações entre os profissionais e usuários como também as relações intraequipe, como relações democráticas, que estimulam a participação, autonomia e decisão coletiva. O acolhimento como técnica é a utilização do saber profissional para produzir respostas ao usuário. Quando se trata da equipe multiprofissional, há a articulação dos diferentes saberes, buscando superar a fragmentação na busca de construção de novas respostas às demandas emergentes. E o acolhimento, na perspectiva da reorientação do serviço, está no centro da proposta do projeto organizacional, que é a base que orienta o trabalho dos profissionais, o processo em equipe, a busca de capacitação. A supervisão é destacada, neste último item, como um importante modo de organização do serviço e de acompanhamento do cotidiano de trabalho assegurando a articulação entre o acolhimento tanto como atitude quanto técnica, o que implicaria uma determinada concepção de clínica para além dos protocolos de triagem.

Os trabalhos reunidos nos Cadernos IPUB n. 17 (2000), sobre o que se convencionou chamar "clínica da recepção", preconizam algumas direções tais como: decantar a demanda no sentido de não atender rapidamente ao pedido inicial; relativizar o pedido de medicação, oferecendo também outras possibilidades; priorizar o diagnóstico da situação sem abandonar o diagnóstico psicopatológico, entendendo que o primeiro de algum modo inclui o segundo. Essa ideia remete à possibilidade de convocar o sujeito a participar do processo de tratamento, deixando de ser tomado apenas como um depósito de sintomas a serem extirpados pelos profissionais (e pela medicação) e tornando-se um agente na compreensão desse processo, adquirindo poder de conduzi-lo através dos profissionais.

- Acompanhamento: é um dos maiores desafios do trabalho na saúde mental. Juntamente com os eixos acesso e acolhimento, o acompanhamento é visto como um analisador da qualidade da assistência. Goldberg (1996) afirma que, dada a reabilitação psicossocial como um processo, o serviço tem que prestar $a$ continuidade do cuidado para que seus objetivos sejam possíveis. Não se trata de uma novidade no campo, e sim de um princípio fundamental do cuidado. $\mathrm{O}$ que se coloca como questão de fundo é como o CAPS acompanha a trajetória de vida dos usuários, através de quais práticas assistenciais o acompanhamento do tratamento é realizado, e como se efetiva algum vínculo entre a clientela e a equipe. 
Para tentar responder a algumas dessas questões, inicialmente cabe distinguir que tipo de acompanhamento é requerido no percurso dos usuários em diferentes situações, e com que recursos. É importante frisar que esse eixo aparece como um indicador durante as reuniões de equipe, ou seja, os próprios profissionais apontaram o acompanhamento como aquilo que imprime qualidade à assistência. Mais do que procurar no cotidiano do trabalho em equipe novos indicadores (satisfação ou autonomia), ou ainda verificar os indicadores epidemiológicos clássicos (número e período de internação), essa pesquisa aponta a possibilidade de fazer valer aquilo que a própria equipe afirma como indicador apropriado de qualidade: nesse caso, o acompanhamento.

A partir dos desdobramentos da pesquisa desenvolvida no interior das reuniões da equipe em supervisão, destacamos um caso em que essa articulação entre os três eixos, com prioridade para o acompanhamento, nos traz novas questões e propostas.

\section{Como sustentar o acompanhamento no CAPS?}

Um rapaz de 23 anos chega ao CAPS com um encaminhamento do Hospital de Custódia, ${ }^{3}$ com o pedido de "agilizar seus documentos e realizar atividades terapêuticas". Ele teve uma briga na escola, ocasião em que "pegou faca" e também havia ameaçado vizinhos, situações que culminaram em uma audiência no juizado da infância e juventude. Lá "teve uma crise", quebrando os computadores da sala do juizado, o que acarretou em um ano e dois meses de "prisão-internação". Ele chega ao CAPS com sua mãe e é acolhido.

Esse rapaz passou a maior parte de sua vida entre instituições e a casa da mãe, esteve inclusive na antiga Febem, ${ }^{4}$ chegou a morar com mais de uma família diferente e viveu em abrigos. Já trabalhou como pescador, ganhou dinheiro em sinal de trânsito e vendeu doces pela cidade. Teve uma irmã que faleceu ainda bem criança, quando os dois moravam na "casa de uma senhora". Há um outro irmão que faleceu adulto, depois de passar anos vivendo na rua. Tem ainda um irmão mais novo que ele, por quem já ficou responsável por este irmão em situações de trabalho nas ruas.

3. O Hospital de Custódia equivale ao Manicômio Judiciário e recebe pacientes com transtorno mental que infringiram a lei.

4. Febem - Fundação Estadual do Bem-Estar do Menor, foi absorvida pelo complexo de instituições para o atendimento de jovens em regime de privação de liberdade, hoje ligado ao Degase. 
Seu pai passou muitos anos internado em um hospital psiquiátrico no estado de Minas Gerais, com poucos momentos fora do hospital. Há alguns anos, provavelmente por conta do processo de fechamento de instituições manicomiais em Minas Gerais, "teve alta" e foi colocado por sua irmã em um asilo para idosos, pago com dinheiro de um benefício social. O paciente já fez várias viagens a Minas Gerais. Chegou a se internar no mesmo hospital do pai, tentou morar com a tia, conseguiu trabalho na colheita de café, enfim, fez várias tentativas de ficar perto do pai.

Sua mãe, segundo a equipe, "não ajuda muito", havendo dificuldade em conversar com ela. A equipe afirma que ela aparece raramente no serviço, mesmo tendo sido convocada a participar do tratamento em várias situações (visitas domiciliares, atendimentos individuais, visitas conjuntas durante a internação do paciente etc.). Inúteis convites, tentativas frustradas de sua inserção no serviço. A participação que os profissionais esperam da mãe no tratamento vai além de idas ao serviço. Inclui ações que poderiam melhorar a situação social e subjetiva do paciente e também dela própria como, por exemplo, a obtenção de documentação pessoal para dar início ao processo de solicitação de benefícios sociais, ou uma melhor orientação de como lidar com ele.

Um profissional narra o percurso do paciente no CAPS e insiste em dizer que é de "difícil adesão", pois já lhe foram feitas várias propostas com mudanças em seu projeto terapêutico, e ele "não cumpre nenhuma". Quando consegue participar de alguma atividade, "dura pouco tempo". Ele diz que quer "pintar quadros" e que "tem de ser em tela", não aceitando os papéis e as tintas comuns. Sua presença no serviço é bastante irregular, assim como o uso da medicação. Ele nem sempre fica na casa de sua mãe, às vezes é abrigado em igrejas, eventualmente fica também na casa da avó. Sua casa não tem telefone e o contato com a mãe é feito através de seu trabalho como faxineira em casas diferentes. Os profissionais relatam que realizam visitas sempre que possível.

Nesse período de um ano e meio, houve duas internações que envolveram o CAPS e mais duas em Minas Gerais, estas últimas em situações nas quais o paciente consegue dinheiro com um pastor de Igreja evangélica para viajar e é internado por algum membro da família.

Desde a chegada do paciente ao serviço, a questão de um "lugar para ficar" e de "pedidos de cuidado" são constantes, segundo o relato da equipe. No começo, estava vivendo com a mãe, o padrasto e o irmão, em uma casa de apenas um cômodo, em situação bastante precária. Nessa casa, ele tinha brigas constantes com o padrasto, já tendo saído de casa várias vezes. Em algumas situações, ameaçou vizinhos e jogou pedras sobre os telhados. Ele faz pedidos constantes à equipe de morar num abrigo, para sair de casa. 
A questão do "lugar" deste paciente é discutida na reunião de supervisão e é considerada como parte de sua história de vida e, portanto, chave para localizarmos um ponto importante de sua determinação subjetiva, o que constitui seu sintoma. A questão do "lugar" é algo que diz respeito a sua própria condição, a partir do diagnóstico de esquizofrenia, como alguém "sem lugar", sua errância reafirma isso. Seus pedidos de internação são interpretados pela equipe como constantes "pedidos de cuidado" e de abrigo.

A equipe discute sobre como conduzir o caso, considerando que ele vem ao serviço e usa medicação de maneira irregular, apesar de entender que medicar é o "tratamento", pede constantemente internação e muitas vezes não aceita as propostas feitas no CAPS. Ao longo da discussão vai se traçando uma maneira de ir "ampliando" o que pode ser entendido como tratamento pelo próprio paciente, para ir além do binômio medicar-tratar. Tentar criar mais vínculos com outros profissionais de modo a ampliar a referência e produzir um "lugar simbólico" mais do que físico no CAPS.

É apontado ainda pela supervisora que um esquizofrênico com sua história de institucionalização não vai necessariamente "cumprir o projeto", nem dias nem horários, nem atividades predeterminadas e que, portanto, a equipe tem que trabalhar com ele sempre que for ao serviço, independentemente do agendamento. É apontado também que nem tudo que o serviço lhe oferece está sendo reconhecido por ele como tratamento, então deve-se trabalhar nesta linha, "próximo ao paciente", para delinear uma maneira de abordá-lo e seguir o seu "estilo" na construção do projeto terapêutico.

Um dia mostrou para uma profissional uma faca na bermuda, dentro da sala em que estava sendo atendido. Ela lhe pediu a faca, pois "não poderia ficar com ela", e ele a entregou tranquilamente. Ele identificou esse ato como estar sendo tratado. Portanto, ser tratado é também ter um "lugar" frente ao outro que intervém no sentido de acolhê-lo e protegê-lo de seus próprios atos, deixando a internação como uma alternativa em situações extremas.

A equipe discute como manejar essas situações e conclui que ele próprio anuncia seus rompantes e que os profissionais sabem reconhecer esse momento, não sendo necessário "segurá-lo" fisicamente.

A discussão segue ressaltando que é importante "estar atento" e que a equipe tem "recursos" para lidar com ele, a partir de uma "relação de confiança", conforme apontou a supervisora. Foi também observado que muitos profissionais falaram sobre o paciente de modo a contribuir para uma melhor abordagem do caso e para a definição de diferentes estratégias e táticas no tratamento. De todos os casos discutidos, este foi o que mais suscitou a participação das diferentes categorias profissionais. Isso demonstra uma real apropriação do caso pelo conjunto da equipe, tendo o acompanhamento como seu maior desafio. 
Um ponto importante a se destacar desse exemplo é a questão de quando e como internar um paciente. A equipe fala do "mal-estar" de ter que decidir pela internação, principalmente quando é um paciente que já se trata no serviço. O problema seria não poder acompanhar o paciente nesse período. É diferenciada a situação em que se utiliza a internação como um último recurso para lidar com a crise, da situação em que se realiza internação "porque o serviço não está disposto a lidar com a crise". Esta segunda situação é apontada como um fracasso do trabalho de CAPS.

Nesse momento, a fala da supervisora esclarece que internar, no limite, às vezes é necessário. O importante é saber decidir o momento da internação e acompanhá-la, para que seja mais breve e bem-sucedida. O que é uma internação bem-sucedida? É poder ter acesso a esse sujeito justamente durante a internação, quando ele pode aceitar melhor nossa presença, de modo que o caso não se perca e o contato seja mantido com o CAPS. Portanto, a internação é acompanhada justamente para se poder trabalhar o retorno.

Esse caso demonstra de modo contundente que na dimensão do acesso atrelado ao acolhimento aparece a inconstância do paciente (suas idas e vindas irregulares, sua convocação insistente à internação, suas escapadas) como um desafio para a equipe na tomada de responsabilidade sobre o caso. E revela que o acompanhamento é crucial para manter o vínculo do paciente com o serviço, incluindo aí o período de internação que deve ser o mais curto possível, de modo a assegurar a continuidade do cuidado e abrir novas possibilidades para o tratamento.

\section{Para concluir}

O acesso e o acolhimento estão imbricados, pois é o desenho do serviço que potencializa ou não esse acesso, e o modo como o acolhimento é feito é determinante para os desdobramentos do atendimento. Em outras palavras, acolher é um processo que depende não só da estrutura ou de aspectos físicos do acesso, mas dos recursos clínicos da equipe, como atender, escutar, avaliar e discriminar as demandas. O acolhimento exige, por um lado, uma ação imediata (urgência) e, por outro, um intervalo de tempo para a resposta (traçar a conduta). Este é o momento de um diagnóstico da situação, de uma primeira abordagem do que se apresenta.

Do acolhimento ao acompanhamento se põe em marcha de fato a tomada de responsabilidade do serviço, que se realiza a cada vez por cada profissional num trabalho partilhado. O acolhimento se desdobra no encaminhamento ou na inclusão do paciente no serviço, e a tomada de responsabilidade é decisiva para o se- 
guimento (follow up do encaminhamento) ou o acompanhamento (continuidade do cuidado).

O caso apresentado acima destaca o acompanhamento, com ênfase nos períodos de internação, que se revela fundamental para uma possível mudança de estratégia na condução do caso. O CAPS sustenta todas as idas e vindas inconstantes do paciente, e o caso é partilhado envolvendo uma parceria de profissionais na referência e também na busca de alternativas para a internação.

Convém observar que o acompanhamento é um recurso de continuidade do cuidado que também se dá de forma descontínua e intermitente, e depende do ritmo de trabalho da equipe, das condições materiais do serviço e da rede e, sobretudo, do manejo de situações e acontecimentos inesperados. É nesse ponto crucial que a supervisão vem cumprir sua função de suporte do trabalho. Como um dispositivo "a mais", enlaça a equipe e convoca a sua responsabilidade no cotidiano, mantendo a troca de experiências e informações para elaborar estratégias de ação em cada caso e a cada passo do acompanhamento.

A supervisão funciona como disparador de questionamentos e aglutinador de propostas e soluções que só podem ser provisórias, contingentes, de acordo com os acontecimentos, mas imprimindo uma direção ao trabalho. Essa direção se dá a partir do que o paciente apresenta como seus movimentos e recursos subjetivos para enfrentar sua condição. Esses são os elementos com os quais podemos proceder à construção do caso, ${ }^{5}$ etapa fundamental do processo para reorientar a clínica imprimindo qualidade ao acompanhamento. Acompanhar e construir o caso são ações intercambiáveis, e uma dá suporte à outra. Desse modo, o acompanhamento se reafirma como um importante indicador da avaliação da qualidade para os CAPS, ao mesmo tempo que é um balizador no cotidiano do trabalho em equipe.

\section{Referências}

A CLínICA da recepção nos dispositivos de Saúde Mental. Cadernos IPUB, Rio de Janeiro, v. VI, n. 17, abr. 2000.

Donabedian, A. Aspects of Medical Care Administration. Boston: Harvard University Press, 1973.

5. A construção do caso se dá em etapas, de modo parcial, no cotidiano do trabalho, recolhendo os elementos advindos das falas e ações do sujeito, e deve ser um exercício coletivo permanente que se realiza através do dispositivo da supervisão. Para detalhes da construção do caso em equipe, ver Figueiredo (2004 e 2005). 
Figueiredo, A. C. A construção do caso clínico: uma contribuição da psicanálise à psicopatologia e à saúde mental. Revista Latinoamericana de Psicopatologia Fundamental, São Paulo, v.VII, n. 1, p. 75-86, mar. 2004.

. Uma proposta da psicanálise para o trabalho em equipe na atenção psicossocial. MENTAL - Revista de Saúde Mental e Subjetividade da UNIPAC/MG, Barbacena, MG, ano III, n. 5, p. 43-55, nov. 2005.

Goldberg, J. Reabilitação como processo - O Centro de Atenção Psicossocial. In: Pitta, A. M. F. Reabilitação psicossocial no Brasil. São Paulo: Hucitec, 1996.

Schmidt, M.B. Avaliação da qualidade de serviços de Saúde Mental: um estudo de caso do CAPS Profeta Gentileza. 2007. 450p. Tese (Doutorado em Saúde Coletiva), Instituto de Medicina Social, Universidade do Estado do Rio de Janeiro.

Silva JR., A. G.; Mascarenhas, M. T. M. Avaliação da atenção básica em saúde sob a ótica da integralidade: aspectos conceituais e metodológicos. In: PinHeIro, R.; Mattos, R. (Org.). Cuidado: as fronteiras da integralidade. Rio de Janeiro: IMSUERJ, 2004. p. 241-257.

\section{Resumo}

(Acceso, recepción y acompañamiento: tres desafíos para el cotidiano de la clinica en Salud Mental)

El presente artículo toma la evaluación de la cualidad de los servicios de salud mental como un instrumento permanente de reflexión sobre la clínica realizada en ellos que permite a partir de lo que aparece durante o a posteriori cualificar desde dentro el trabajo de atención psicosocial, más allá de un proyecto de evaluación a priori hecho desde fuera. Señalamos ejes del campo da avaluación que pueden ser aplicados tanto en el análisis como en la organización de lo cotidiano de la clínica en los CAPS. Proponemos los ejes, acceso, recepción y acompañamiento como elementos fundamentales para el análisis de la asistencia y para la calificación permanente de los servicios. Presentamos aquí parte de un caso de tenor evaluativo realizado no CAPS Profeta Gentileza - SMS-Rio en el año 2006, como tesis de doctorado de una de las autoras, que obtuvo material de los casos a través del relato gravado en reunión de control del equipo en el servicio. El control fue elegido como local privilegiado de investigación por ser considerado un dispositivo fundamental para el funcionamiento del servicio, la organización y la dinámica del trabajo en equipo, y funcionar como un evaluador permanente de lo cotidiano de un CAPS.

Palabras claves: Atención psicosocial, evaluación de servicios, salud mental, supervision 
(Accès, accueil et accompagnement: trois défis du quotidien de la clinique en Santé Mentale)

Cet article est basé sur l'évaluation de la qualité des services de santé mentale comme instrument permanent de réflexion sur la clinique de ces services pour, d'une part, un projet d'évaluation réalisé a priori de façon externe et, d'autre part, pour qualifier de façon interne le travail du service psychosocial à partir de ce qui surgit durant ou a posteriori. Nous indiquons les axes du domaine évalué qui peuvent être appliqués non seulement à l'analyse, mais aussi à l'organisation du quotidien de la clinique des CAPS. Nous proposons les axes accès, accueil et accompagnement comme éléments fondamentaux à l'analyse de l'assistance, ainsi que pour la qualification permanente des services. Nous présentons ici une partie d'une étude de cas de nature évaluative réalisée au "CAPS Profeta Gentileza-SMS-Rio» en 2006 qui fait partie de la thèse de doctorat de l'une des auteures. Le matériel des cas a été obtenu à travers les rapports enregistrés des réunions de supervision d'équipes en service. La supervision a été choisie comme locus privilégié de la recherche, car elle est considérée un dispositif fondamental au fonctionnement du service, l'organisation et la dynamique du travail en équipe et fonctionne comme estimateur permanent du quotidien du CAPS.

Mots-clés: Soins psychosociaux, évaluation des services, santé mentale, supervision

(Access, interviews and accompaniment: three challenges for day-to-day work at a mental health clinic)

The present article discusses the evaluation of the quality of the services rendered at mental health centers (CAPS) and sees such evaluation as a permanent instrument for reflecting on the centers' activities. Evaluation therefore goes beyond the concept of a view from outside. In this case, it consists of a view from inside, while work is being carried out or a posteriori. Lines of evaluation, which can be applied to analyses and to the organization of everyday routines at such centers, are also discussed. The author suggests three areas of activities that should be evaluated: access, first interviews and accompaniment, as these are considered fundamental aspects of the work carried out at CAPS. Part of a case study of evaluation for a doctoral thesis written in 2006 is presented here. The case material was collected during supervision meetings with the staff, a setting that is considered an essential activity for analyzing the organization and dynamics of the work carried out at CAPS. From this perspective, supervisions serve as permanent evaluators of everyday activities at CAPS.

Key words: Psychosocial attention, evaluation of services, mental health, supervision

Citação/Citation: SchmidT, M.B.; FigueIREdo, A.C. Acesso, acolhimento e acompanhamento: três desafios para o cotidiano da clínica em Saúde Mental. Revista Latinoamericana de Psicopatologia Fundamental, São Paulo, v. 12, n. 1, p. 130-140, mar. 2009. 
Editor do artigo/Editor: Profa. Dra. Ana Cristina Figueiredo.

Recebido/Received: 6.2.2009 / 2.6.2009 Aceito/Accepted: 9.2.2009/ 2.9.2009

Copyright: () 2009 Associação Universitária de Pesquisa em Psicopatologia Fundamental/ University Association for Research in Fundamental Psychopathology. Este é um artigo de livre acesso, que permite uso irrestrito, distribuição e reprodução em qualquer meio, desde que $\mathrm{o}$ autor e a fonte sejam citados/This is an open-access article, which permits unrestricted use, distribution, and reproduction in any medium, provided the original author and source are credited.

Financiamento/Funding: Os autores declaram não ter sido financiados ou apoiados/The authors have no support or funding to report.

Conflito de interesses: Os autores declaram que não há conflito de interesses/The authors declare that they have no conflict of interest.

\section{Moema Belloni Schmidt}

Doutora em Saúde Coletiva pelo Instituto de Medicina Social - IMS-Universidade estadual do Rio de Janeiro; membro da equipe de assessoria técnica da Coordenação Nacional de Saúde Mental do Ministério da Saúde, Brasília-BR.

SQN 203 bloco D apto. 503

70833-040 Brasília, DF, Brasil

e-mail: moemabschmidt@gmail.com

\section{Ana Cristina Figueiredo}

Doutora em Saúde Coletiva pelo Instituto de Medicina Social - IMS-Universidade Estadual do Rio de Janeiro - UERJ (Rio de Janeiro, RJ, Brasil); Professora Associada do Instituto de Psiquiatria - IPUB-Universidade Federal do Rio de Janeiro - UFRJ (Rio de Janeiro, RJ, Brasil); Supervisora de CAPS e da rede de saúde mental do Município do Rio de Janeiro em parceria com a SMS-Rio.

Rua Smith de Vasconcelos, 55/101

22241-160 Rio de Janeiro, RJ, Brasil

e-mail: anacrisfigueiredo@gmail.com 